Brief Report

\title{
Spontaneous Curvature Induced Stretching-Bending Mode Coupling in Membranes
}

Efim I. Kats

check for

updates

Citation: Kats, E.I. Spontaneous Curvature Induced Stretching-

Bending Mode Coupling in

Membranes. Physics 2021, 3, 367-371.

https://doi.org/10.3390/

physics3020025

Received: 15 April 2021

Accepted: 10 May 2021

Published: 14 May 2021

Publisher's Note: MDPI stays neutral with regard to jurisdictional claims in published maps and institutional affiliations.

Copyright: (C) 2021 by the author. Licensee MDPI, Basel, Switzerland. This article is an open access article distributed under the terms and conditions of the Creative Commons Attribution (CC BY) license (https:/ / creativecommons.org/licenses/by/ $4.0 /)$.
Landau Institute for Theoretical Physics, RAS, 142432 Chernogolovka, Moscow Region, Russia; kats@itp.ac.ru

\begin{abstract}
In this paper, a simple example to illustrate what is basically known from the Gauss' times interplay between geometry and mechanics in thin shells is presented. Specifically, the eigen-mode spectrum in spontaneously curved (i.e., up-down asymmetric) extensible polymerized or elastic membranes is studied. It is found that in the spontaneously curved crystalline membrane, the flexural mode is coupled to the acoustic longitudinal mode, even in the harmonic approximation. If the coupling (proportional to the membrane spontaneous curvature) is strong enough, the coupled modes dispersions acquire the imaginary part, i.e., effective damping. The damping is not related to the entropy production (dissipation); it comes from the redistribution of the energy between the modes. The curvature-induced mode coupling makes the flexural mode more rigid, and the acoustic mode becomes softer. As it concerns the transverse acoustical mode, it remains uncoupled in the harmonic approximation, keeping its standard dispersion law. We anticipate that the basic ideas inspiring this study can be applied to a large variety of interesting systems, ranging from still fashionable graphene films, both in the freely suspended and on a substrate states, to the not yet fully understood lipid membranes in the so-called gel and rippled phases.
\end{abstract}

Keywords: membranes; vibration modes

\section{Introduction}

It is my pleasure and honor to present my work in the Special Issue of journal of Physics, dedicated to the 70th birthday of Prof. Mikhail Tribelsky. Many years ago, we collaborated with Misha (as I became used to calling him), investigating phase transitions in biaxial liquid crystals. Misha came up with the idea of our joint article and guided me through the long process of writing. The paper I am presenting now (also from the realm of soft matter physics) is a small token of my gratitude and respect to Misha Tribelsky.

The study is motivated by a recent influential paper [1], where the authors found that even in the harmonic approximation, in-plane and out-of-plane vibrations of a lowdimension $(D=1,2)$ elastic system can be coupled. The harmonic coupling in Ref. [1] occurs if the stress-free state of the membrane is curved. Provided that the "up-down" symmetry of the system is not broken, the only way to couple the modes is related to the Gaussian curvature, which is symmetric (even) with respect to "up-down" inversion; for more details, see monographs and review [2-6] or original papers [7-10]. However, in many realistic and experimentally relevant situations, the "up-down" symmetry is spontaneously broken [3,5]. Moreover, it can be nonuniformly broken over the membrane surface, e.g., due to asymmetric adsorption of different molecular species. In such a situation, the up-down non-symmetric free energy in the harmonic approximation can be written in terms of the scalar out-of-plane displacement $f$ and the $2 D$ vector $\mathbf{u}$ of the in-plane displacements: $[2,3,5]$

$$
F_{\text {elastic }}=\int d^{2} r\left[\frac{1}{2} \kappa\left(\nabla^{2} f\right)^{2}+C_{0}(r) \nabla^{2} f+\frac{1}{2} \lambda u_{i i}^{2}+\mu\left(\frac{\partial u_{i}}{\partial x_{j}}+\frac{\partial u_{j}}{\partial x_{i}}\right)^{2}\right],
$$


where $\kappa$ is the bending (curvature) elastic modulus, the subscripts $i$ and $j$ take on the values 1,2 for the Cartesian axis within the membrane plane (in the limit of small displacements, the topological Gaussian curvature contribution is neglected here), $\mu$ and $\lambda$ are the stretching Lame coefficients, and $C_{0}(r)$ is non-uniform over the membrane spontaneous curvature. In the linear approximation, the only term allowed by symmetry is

$$
C_{0}=\gamma_{\text {int }} \operatorname{div} \mathbf{u}
$$

where $\gamma_{\text {int }}$ is the curvature-induced mode coupling coefficient.

\section{Basic Derivation}

With the free energy expansion (1) one can find the eigen-modes of the system. Since the intent of this study is to investigate dynamic behavior of spontaneously curved membranes, first of all, the free energy (1) has to be supplemented by the kinetic energy terms related to the in-plane and out-of-plane displacements dynamics. As a result, one gets the dynamic action,

$$
S=\frac{1}{2}\left(\frac{\partial f}{\partial t}\right)^{2}+\frac{1}{2}\left(\frac{\partial u_{i}}{\partial t}\right)^{2}+F_{\text {elastic }}
$$

where, for the sake of simplicity, the units with the mass density $\rho=1$ are used, and $t$ is the time. Then, in the Fourier space, the corresponding Euler-Lagrange equations for the coupled eigen-modes read:

$$
\begin{aligned}
\omega^{2} \tilde{f}-\kappa q^{4} \tilde{f}-i \gamma_{\mathrm{int}} q^{2} q_{i} \tilde{u}_{i} & =0, \\
\omega^{2} \tilde{u}_{i}-i \gamma_{\mathrm{int}} q_{i} q^{2} \tilde{f}-\mu q^{2} \tilde{u}_{i}-\mu q_{i}\left(q_{j} \tilde{u}_{j}\right)-\lambda q_{i}\left(q_{m} \tilde{u}_{m}\right) & =0,
\end{aligned}
$$

where $\tilde{f}$ and $\tilde{\mathbf{u}}$ are the Fourier transforms of the displacements

$$
f(\mathbf{r}, t)=\int \frac{d^{2} q}{(2 \pi)^{2}} \frac{d \omega}{2 \pi} \exp (-i \omega t+i \mathbf{q r}) \tilde{f}(\mathbf{q}, \omega),
$$

and similarly for the in-plane displacements $u_{i}$.

From Equation (4), one can see that the out-of-plane displacement is coupled to the only longitudinal component (with respect to the wave-vector) of the in-plane displacements, similarly to the case of up-down symmetric crystalline membranes considered in Ref. [11]. Therefore, it is convenient to express the in-plane displacements in terms of the longitudinal, $u_{l}$, and transverse, $u_{t}$, components, namely,

$$
u_{i}=u_{l} \frac{q_{i}}{q}+u_{t} \epsilon_{i j} \frac{q_{j}}{q}
$$

where $\epsilon_{i j}=-\epsilon_{j i}$ is the antisymmetric second-rank tensor. Then, the equations of motion can be rewritten as:

$$
\begin{aligned}
\omega^{2} \tilde{f}-\kappa q^{4} \tilde{f}-i \gamma_{\mathrm{int}} q^{3} \tilde{u}_{l} & =0, \\
\omega^{2} \tilde{u}_{l}-i \gamma_{\mathrm{int}} q_{i} q^{2} \tilde{f}-(2 \mu+\lambda) q^{2} \tilde{u}_{l} & =0, \\
\omega^{2} \tilde{u}_{t}-\mu q^{2} \tilde{u}_{t} & =0 .
\end{aligned}
$$

\section{Eigen-Modes in Spontaneously Curved Membranes}

From Equation (6), one can see that the transverse acoustic mode, which is decoupled from flexural mode in the harmonic approximation, has the standard dispersion law:

$$
\omega_{t}^{2}=\mu q^{2} .
$$


In the harmonic approximation, the dispersion laws for the two coupled (in the spontaneously curved membrane) longitudinal acoustic and flexural modes are:

$$
\omega_{1,2}^{2}=\frac{\kappa q^{4}+(\lambda+2 \mu) q^{2}}{2} \pm\left[\left(\frac{\kappa q^{4}-(\lambda+2 \mu) q^{2}}{2}\right)^{2}-\gamma_{\text {int }}^{2} \kappa^{2} q^{5}\right]^{1 / 2} .
$$

This expression is the main result of this paper and is ready for further inspection. From Equation (8), one arrives at the two following conclusions.

- For the sufficiently strong spontaneous curvature-induced coupling, there is an interval of the wave vectors,

$$
\frac{(\lambda+2 \mu)^{2}}{\kappa^{2} \gamma_{\text {int }}^{2}}<q<\sqrt{\frac{\lambda+2 \mu}{\kappa}},
$$

where the coupled-modes dispersions acquire the imaginary part, i.e., effective damping. The damping is not related to the entropy production (dissipation), since there is no any dissipative term in the action (3). The damping occurs from the redistribution of the energy of the modes.

- In the limit of weak coupling,

$$
\gamma_{\text {int }}^{2} \kappa^{2} q^{5}<\frac{\left(\kappa q^{4}-\mu q^{2}\right)^{2}}{4}
$$

the coupled modes remain purely propagating, and their dispersion laws read:

$$
\begin{array}{r}
\omega_{1}^{2}=\kappa q^{4}+\frac{\gamma_{\text {int }}^{2} \kappa^{2}}{\lambda+2 \mu} q^{3}, \\
\omega_{2}^{2}=(2 \mu+\lambda) q^{2}-\frac{\gamma_{\text {int }}^{2} \kappa^{2}}{(2 \mu+\lambda)} q^{3} .
\end{array}
$$

Therefore, the curvature-induced mode coupling makes the flexural mode more rigid, and the acoustic mode becomes softer.

\section{Outlook and Conclusions}

To summarize, in this paper, the eigen-mode spectrum in spontaneously curved, i.e., up-down asymmetric, extensible polymerized or elastic membranes is calculated. It is found that in the spontaneously-curved crystalline membrane, the flexural mode is coupled to the acoustic longitudinal mode, even in the harmonic approximation. If the coupling, being proportional to the membrane spontaneous curvature, is strong enough, the coupledmode's dispersions acquire the imaginary part, i.e., effective damping. The damping is not related to the entropy production (dissipation): it comes from the redistribution of the energy of the modes. The curvature-induced mode coupling makes the flexural mode more rigid, and the acoustic mode becomes softer. What concerns the transverse acoustical mode, it remains uncoupled in the harmonic approximation, keeping its standard dispersion law.

Let us close with some conclusions about where the results presented here can be applied. First, one may think about the famous graphene films [12-16] either on substrate or freely suspended. In both cases, experimental observations suggest that the graphene film becomes spontaneously corrugated. Although the main physical phenomena leading to the graphene film corrugations are not yet fully understood (see, e.g., Refs. [17-19]), the conclusion of the very existence of the corrugated state seems inescapable. The second system one might have in mind is a rippled state of lipid membranes, below so-called main phase transition [4-6]. The macroscopic structure and physical properties of the rippled gel state are still debated in the literature; see, e.g., Refs. [20,21]. The simple calculations, presented in this paper, are a step forward to check whether the state is liquid, liquid- 
crystalline (liquid layers), or crystalline (with a positional order within the layer). Analysis of eigen-mode spectra provides a hint to disentangle both states.

It is worth noting that only the mean-field approximation was considered in this paper. However, the membranes are effectively two-dimensional objects (on scales larger than the membrane thickness). Therefore, thermal fluctuations can affect behavior, first of all, renormalizing the membrane elastic moduli. This renormalization of the bending modulus $\kappa$ is well known for liquid-like membranes. It turns out that thermal fluctuations make the liquid-like membrane softer, i.e., reduce the bending modulus $[5,6]$. For crystalline membranes (see Refs. $[9,10]$ and recent studies [22,23]), the bending modulus increases, i.e., the membrane becomes harder, whereas the stretching Lame coefficients $\mu$ and $\lambda$ decrease. Similarly, one can also find the fluctuation renormalization of the spontaneous curvature or, in terms of this study, the curvature-induced mode coupling coefficient $\gamma_{\text {int. }}$. The one-loop approximation calculations $[5,6]$ yield the following scaling law:

$$
\gamma_{\text {int }} \propto(3 T / 4 \pi \kappa)^{-1 / 3}
$$

with $T$ being the temperature, i.e., thermal fluctuations decrease the curvature-induced mode coupling. In this paper, only the surface of this reach subject is stratched. This study deliberately focused on the most limited possible question, which can be answered by calculations "on a back of the envelope". There is a plenty of work ahead.

Acknowledgments: I thank V.V. Lebedev for useful discussions.

Conflicts of Interest: The author declares no conflict of interest.

\section{References}

1. Kernes, J.; Levine, A.J. Effects of curvature on the propagation of undulatory waves in lower dimensional elastic materials. Phys. Rev. E 2021, 103, 013002. [CrossRef] [PubMed]

2. Nelson, D.; Piran, T.; Weinberg, S. (Eds.) Statistical Mechanics of Membranes and Surfaces; World Scientific: Singapore, 1989. [CrossRef]

3. Chaikin, P.M.; Lubensky, T.C. Principles of Condensed Matter Physics; Cambridge University Press: Cambridge, UK, 2000. [CrossRef]

4. Bowick, M.J.; Travesset, A. The statistical mechanics of membranes. Phys. Rep. 2001, 344, 255-308. [CrossRef]

5. Safran, S.A. Statistical Thermodynamics of Surfaces, Interfaces, and Membranes; CRC Press: Boca Raton, FL, USA, 2003. [CrossRef]

6. Kats, E.I.; Lebedev, V.V. Fluctuational Effects in the Dynamics of Liquid Crystals; Springer: New York, NY, USA, 1994. [CrossRef]

7. Helfrich, W. Elastic properties of lipid bilayers: Theory and possible experiment. Z. Naturforsch. 1973, 28, 693-703. [CrossRef] [PubMed]

8. Seifert, U. Configurations of fluid membranes and vesicles. Adv. Phys. 1997, 46, 13-137. [CrossRef]

9. Nelson, D.R.; Peliti, L. Fluctuations in membranes with crystalline and hexatic order. J. Phys. France 1987, 48, 1085-1092. [CrossRef]

10. Aronovitz, J.A.; Lubensky, T.C. Fluctuations of solid membranes. Phys. Rev. Lett. 1988, 60, 2634-2638. [CrossRef] [PubMed]

11. Kernes, J.; Levine, A.J. Geometrically-induced localization of flexural waves on thin warped physical membranes. arXiv 2020, arXiv:2011.07152v1.

12. Novoselov, K.S.; Geim, A.K.; Morozov, S.V.; Jiang, D.; Zhang, Y.; Dubonos, S.V.; Grigorieva, I.V.; Firsov, A.A. Electric field effect in atomically thin carbon films. Science 2004, 306, 666-669. [CrossRef] [PubMed]

13. Novoselov, K.S.; Jiang, D.; Schedin, F.; Booth, T.J.; Khotkevich, V.V.; Morozov, V.V.; Geim, A.K. Two-dimensional atomic crystals. Proc. Natl. Acad. Sci. USA 2005, 102, 10451-10453. [CrossRef] [PubMed]

14. Neto, A.H.C.; Guinea, F.; Peres, N.M.R.; Novoselov, K.S.; Geim, A.K. The electronic properties of graphene. Rev. Mod. Phys. 2009, 81, 109-161. [CrossRef]

15. Vozmediano, M.A.H.; Katsnelson, M.I.; Guinea, F. Gauge fields in graphene. Phys. Rep. 2010, 496, 109-152. [CrossRef]

16. Geim, A.K.; Grigorieva, I.V. Van der Waals heterostructures. Nature 2013, 499, 419-425. [CrossRef] [PubMed]

17. Fasolino, A.; Los, J.H.; Katsnelson, M.I. Intrinsic ripples in graphene. Nat. Mater. 2007, 6, 858-861. [CrossRef]

18. Bao, W.; Miao, F.; Chen, Z.; Zhang, H.; Jang, W.; Dames, C.; Lau, C.N. Controlled ripple texturing of suspended graphene and ultrathin graphite membranes. Nat. Nanotechnol. 2009, 4, 562-566. [CrossRef] [PubMed]

19. Deng, S.; Berry, V. Wrinkled, rippled and crumpled graphene: an overview of formation mechanism, electronic properties, and applications. Mater. Today 2016, 19, 197-213. [CrossRef]

20. Raghunathan, V.A.; Katsaras, J. $L_{\beta}^{\prime} \rightarrow L_{c}$ phase transition in phosphatidylcholine lipid bilayers: A disorder-order transition in two dimensions. Phys. Rev. E 1996, 54, 4446-4449. [CrossRef] [PubMed]

21. Akabori, K.; Nagle, J.F. Structure of the DMPC lipid bilayer ripple phase. Soft Matter 2015, 11, 918-926. [CrossRef] [PubMed] 
22. Burmistrov, I.S.; Gornyi, I.V.; Kachorovskii, V.Y.; Katsnelson, M.I.; Los, J.H.; Mirlin, A.D. Stress-controlled Poisson ratio of a crystalline membrane: Application to graphene. Phys. Rev. B 2018, 97, 125402. [CrossRef]

23. Saykin, D.R.; Kachorovskii, V.Y.; Burmistrov, I.S. Phase diagram of a flexible two-dimensional material. Phys. Rev. Res. 2020, 2, 043099. [CrossRef] 